This item was submitted to Loughborough's Institutional Repository (https://dspace.lboro.ac.uk/) by the author and is made available under the following Creative Commons Licence conditions.

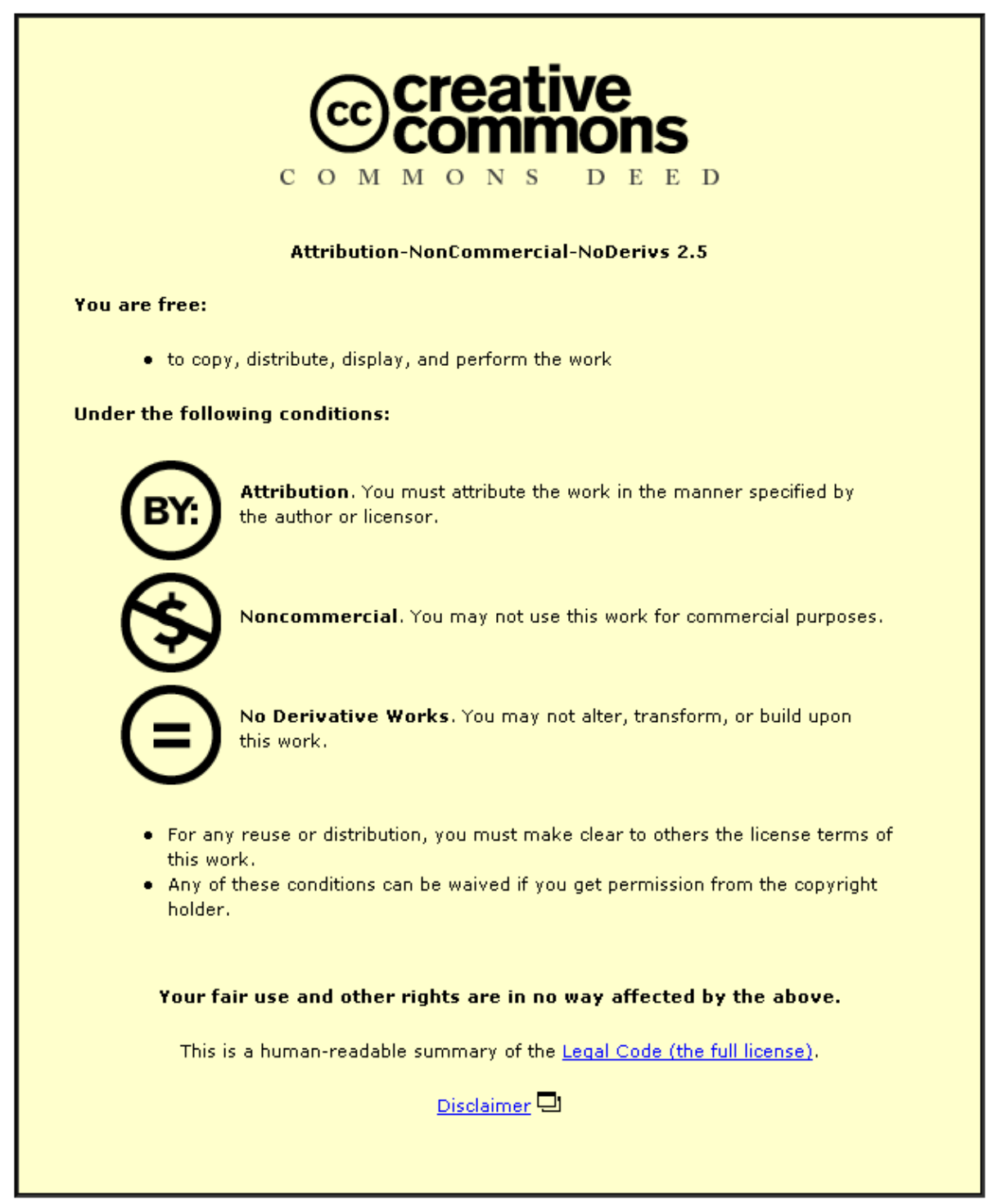

For the full text of this licence, please go to: http://creativecommons.org/licenses/by-nc-nd/2.5/ 


\title{
Polarisation Dependent EBG Surface with an Inclined Sheet Via
}

\author{
Sadiq Ullah, James A. Flint, R. D. Seager \\ Department of Electronic \& Electrical Engineering, Loughborough University \\ Ashby Road, Loughborough, Leicestershire, LE11 3TU, UK. \\ \{S.Ullah, J.A.Flint, R.D.Seager\}@lboro.ac.uk
}

\begin{abstract}
This paper presents a novel polarisation dependent EBG (PDEBG) surface which makes use of sheet vias. The performance of the surface is analysed whilst varying the length, thickness and inclination of the vias. It is observed that the phase difference between the $x$-polarised and $y$-polarised component of the reflected wave can be controlled by varying these parameters. Furthermore, the surface exhibits a polarisation conversion property with potential use in a variety of antennas.
\end{abstract}

\section{INTRODUCTION}

High Impedance Surface (HIS) [1] design is becoming an important discipline in antenna engineering. HISs behave as Electromagnetic Bandgap (EBG) structures ([2], [3]) or Artificial Magnetic Conductors (AMC) [4], depending on the nature of the incident waves in question. EBG structures are used as ground planes in antenna applications for surface wave suppression and in-phase reflection [5], antenna miniaturization [6], and polarisation dependence applications [7]-[13].

In this paper square patch elements are used which are individually shorted to the metal ground plane with a sheet metal vias as an alternative to conventional cylindrical (pin) vias. The geometry addressed by this paper is shown in Fig. 1.

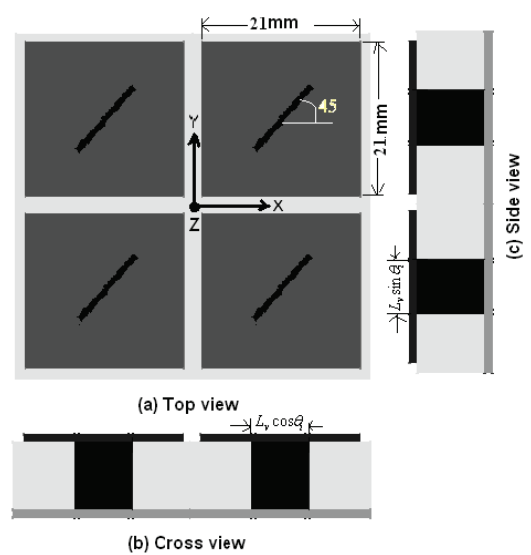

Fig. 1 EBG structure with a $\theta_{v}=45^{\circ}$ inclined sheet via

It will be demonstrated how the length $\left(L_{v}\right)$, thickness $\left(T_{v}\right)$ and inclination angle $\left(\theta_{v}\right)$ of the sheet via affects the reflection phase of the reflected waves and it will be shown how proper selection of these parameters gives polarisation dependent behaviour in some circumstances. Good electric conductors such as metallic ground planes exhibit both frequency and polarisation independent behaviour over a wide frequency band, however the phase inversion experienced by reflections from the surface are problematic in some applications. The emergence of magnetic conductors which can be realised from periodic arrays of patches or similar elements can overcome the phase inversion at the surface and can have other useful properties, albeit over a typically small frequency range. Furthermore, other useful properties have been realised by making use of anisotropic arrays where the periodicity or other parameter is changes in a particular coordinate direction. For example, Yang and Rahmat-Samii [7] have proposed a polarisation dependent EBG (PDEBG) and proved that the reflection phase of the PDEBG surface is a function of frequency and polarisation state of the incident wave. The phase difference between the two components of the reflected wave can be adjusted by modifying the unit cell geometry of the PDEBG [7], i.e. replacing the square patch with a rectangular patch, introducing symmetrical slots in the square patch, and offsetting the metal vias. These techniques are useful tools to obtain polarisation dependent EBG surfaces, but still there is a need to introduce added flexibility without excessive increase in unit cell dimensions, i.e. the ability to fine-tune the reflection phase-difference between the components of the reflected wave over 0-180 degrees. The current paper offers an alternative approach to this problem.

Section II elaborates the design procedure for the polarisation dependent EBG and the reference design used for to compare with the new design. Sections III and IV present the results, and conclusions are made in Section V.

\section{DESIGN PROCEDURE FOR THE REFERENCE EBG}

In order to make the comparisons in this paper, a periodic square patch-based EBG was designed using well established equations [14]. Selecting the resonant frequency $f_{r}=3 \mathrm{GHz}$ yields the following dimensions:

$$
\begin{aligned}
& L=W=0.21 \lambda_{3 \mathrm{GHz}}=21 \mathrm{~mm} \\
& g=0.02 \lambda_{3 \mathrm{GHz}}=2 \mathrm{~mm} \\
& a=W+g=23 \mathrm{~mm} \\
& \mathcal{E}_{r}=4.5, t=1.6 \mathrm{~mm}
\end{aligned}
$$


where the symbols used refer to dimensions in Fig. 2, or otherwise take their usual meaning. A substrate permittivity of 4.5 was selected as the final application of the surface is to be on FR4 material, but there is no special reason for choosing this value. For the sheet via surface, the same dimensions and substrate properties were used however new parameters were required to represent the length of the via, $L_{v}$ the thickness $T_{v}$ and the via inclination angle, $\theta_{v}$. It is these values that form the major part of the analysis in the parametric study.

The reflection phase of the EBG surface is given by [14]:

$$
\theta=\operatorname{Arg}\left(\frac{Z_{s}(\omega)-\eta}{Z_{s}(\omega)+\eta}\right)
$$

where $\eta$ and $Z_{s}(\omega)$ are the impedance of free space and the surface impedance of the EBG surface respectively. It would be expected that any changes in the phase response in the sheet via EBG would be brought about due to changes in $Z_{s}(\omega)$ which is in turn due to the effective per cell surface capacitance, $C$, and inductance, $L$. The following relationship can be derived [14]:

$$
Z_{s}(\omega)=\frac{j \omega L}{1-\omega^{2} L C}
$$

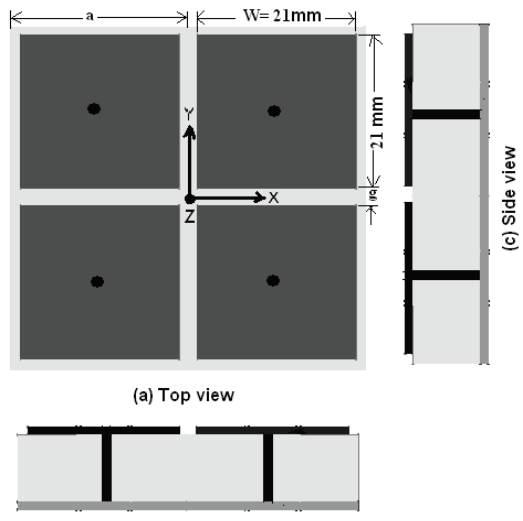

(b) Cross view

Fig. 2 Reference EBG structure employing a cylindrical (pin) via

Consequently, the frequency at which the surface operates as a HIS and where the reflection phase is zero is therefore given by:

$$
\omega_{r}=\frac{1}{\sqrt{L C}}
$$

The capacitance term in this equation is primarily due to fringing between adjacent patches and this remains unchanged in the new surface. The inductance, on the other hand, has been modified significantly by the presence of the thick sheet via. It is not straightforward to express this simply as in the pin via case and in addition anisotropy has been introduced. The following analysis therefore concentrates on numerical simulation of a slanted sheet via of various thicknesses and lengths.

\section{LINEAR EXCITATION RESULTS}

The surfaces were simulated in TLM [15] and postprocessed in Matlab v7.4. In each case the surface was simulated with periodic boundary conditions at the $x$ and $y$ extremities of the unit cell. The mushroom type EBG surface was included as the reference surface for comparison. The first set of simulations involved illuminating the surface with linearly polarised plane waves and analysing the reflection phase. Separate simulations were carried out for $x$ and $y$ polarised waves. In each case the reflected wave was compared in phase with the incident wave in the excited polarisation.

\section{A. Varying the length of the sheet via}

In the first case the inclination and thickness of the sheet via was fixed at $\theta_{v}=0^{\circ}$ and $2 \mathrm{~mm}$ respectively and $L_{v}$ varied from 2-12 $\mathrm{mm}$. The results are shown in Fig. 3. For the case where the length is $2 \mathrm{~mm}$ the reflection phase of both the $x$ and $y$ polarised components of the incident plane wave are identical and as would be expected, the results are extremely similar to that of a cylindrical, small pin via (labelled 'Mushroom' in the plots).

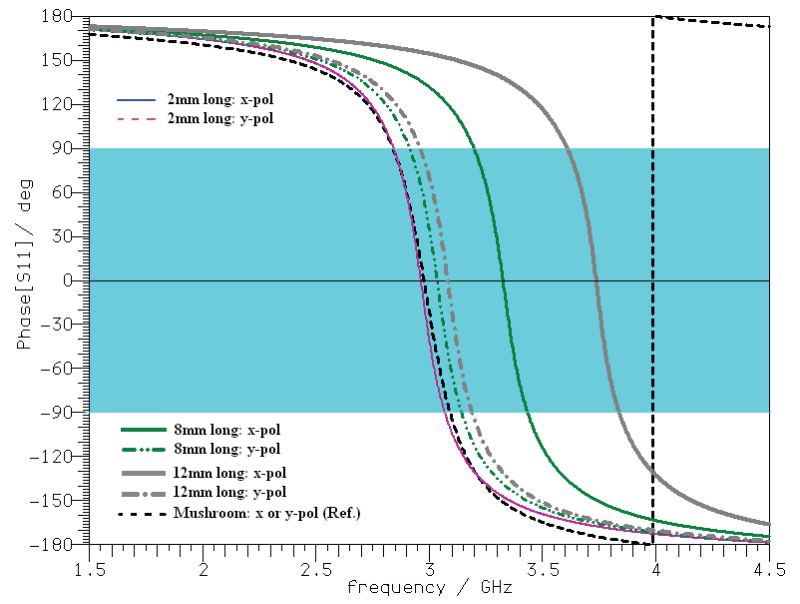

Fig. 3 Reflection phase of the EBG by varying the slot via length, $L_{v}$

When the length of the sheet via is increased, in the direction of the $x$-axis, then it is observed that the reflection phase curve for the $x$-polarised component is shifted towards the right along the frequency axis. In other words the effective inductance presented to the $x$-polarised component is reduced when the length of the sheet via is increased in that direction. This would be expected due to the shorter path length associated with that polarisation.

\section{B. Varying thickness of the sheet via}

In this case the length of the sheet via remains constant (i.e. $8 \mathrm{~mm}$ ), but the thickness is varied from approximately $0.05-$ $2 \mathrm{~mm}$. As the thickness is increased, the reflection curves of the $y$-polarised component of the incident plane wave shifts to 
the right away from $3 \mathrm{GHz}$ point. Therefore the phase difference between the $x$ and $y$ polarisation components can, to some extent, be controlled by varying the thickness of the sheet via. The resonant shift in the reflection phase curve is minimal in this case due to smaller variation in the width of the sheet via (Fig. 4).

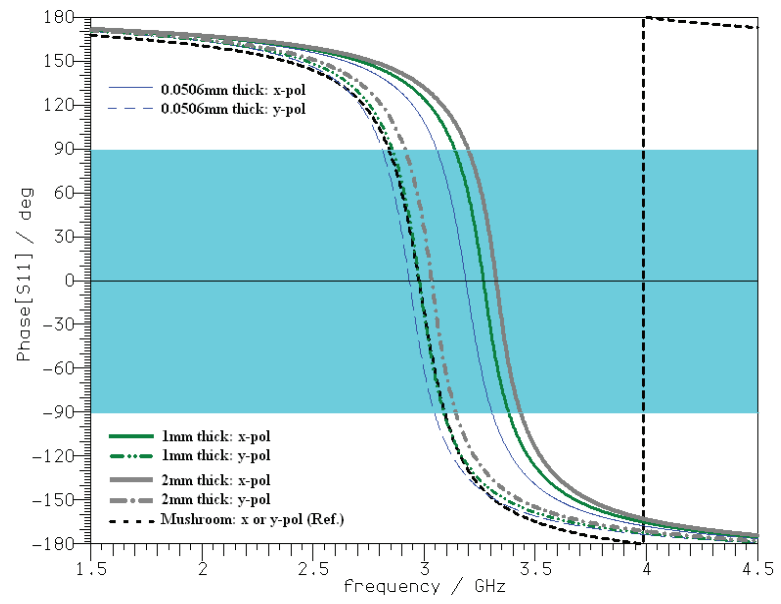

Fig. 4 Reflection phase changes with thickness, $T_{v}$

\section{Varying the inclination of the sheet via}

Finally the inclination of the sheet via is changed and the reflection phase curves of the plane wave are analyzed for the same simulation conditions as shown in Fig. 5.

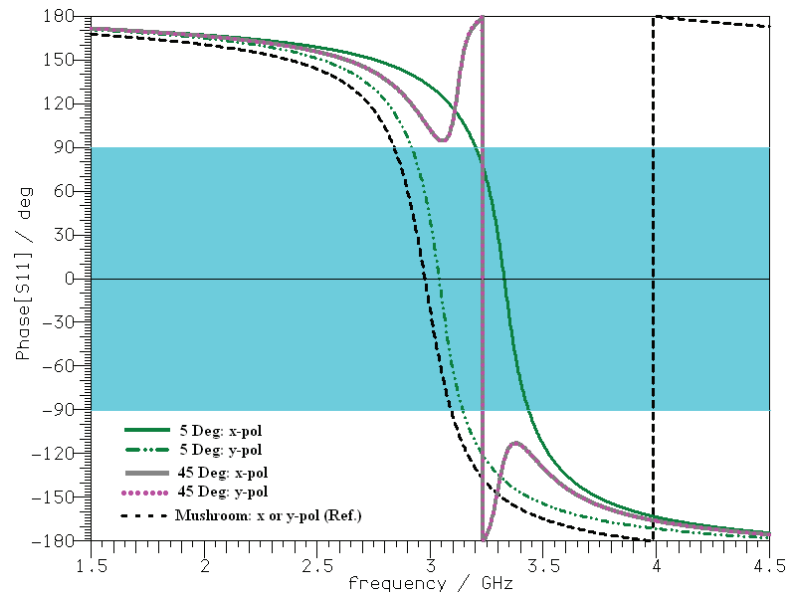

Fig. 5 Reflection phase due to changing via angle, $\theta$

The EBG shows distinctive behavior for the $45^{\circ}$ inclination angle and this was investigated further by using a circularly polarised incident wave and applying more detailed analysis methods on the reflected waves. In the following section the $45^{\circ}$ slanted via is considered.

\section{CirCUlar Polarisation RESUltS}

A pure left-hand circularly polarised (LHCP) plane wave was excited in the simulation at a suitable distance away from the surface. A pair of E-field measurement points were placed in the computer model between the excitation plane and the surface and from these, the forward- and reverse-going wave components were resolved. The amplitudes of the $x$ and $y$ components of the incident electric field are denoted $E_{i x}$ and $E_{i y}$ with their chosen magnitudes being $1 \mathrm{~V} \cdot \mathrm{m}^{-1}$ and their reflected counterparts, $E_{o x}$ and $E_{o y}$.

The field components produced in the simulation are shown in Fig. 6. It can be seen that there are distinct regions of operation which will be explained further.

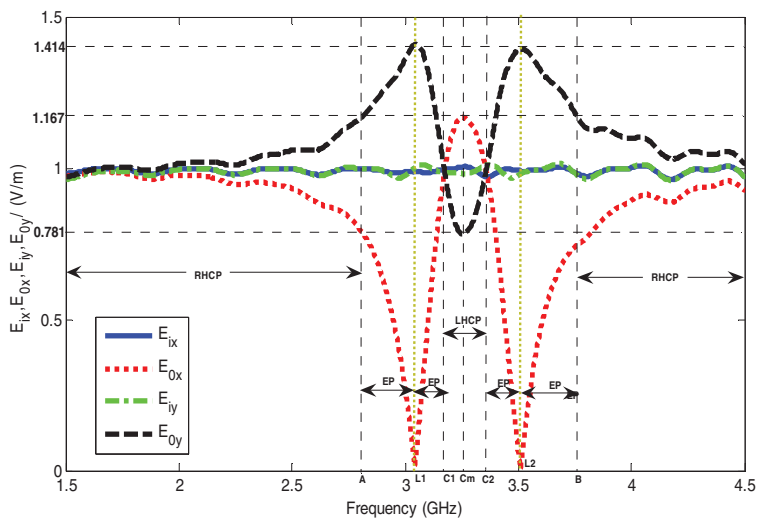

Fig. 6 Ingoing and outgoing waves components for the inclined via EBG

Further analysis was carried out on the relative phase of the reflected waves. This is shown in Fig. 7.

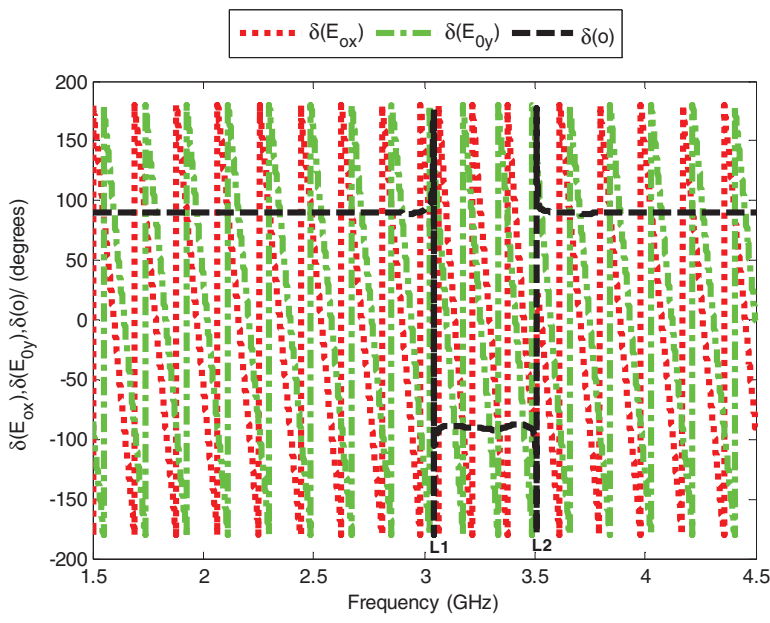

Fig. 7 Phase analysis of the outgoing wave

where $\delta\left(E_{o x}\right), \delta\left(E_{o y}\right)$ are the phase differences experienced by the separate field components at the surface and $\delta(o)$ is the phase difference between the reflected field components. For high and low frequencies the phase difference between the reflected components remains constant at $90^{\circ}$, as would be expected for LHCP incident waves (converted to RHCP, as would take place for an electric conductor), however this flips to $-90^{\circ}$ in the centre band, indicating a change of $\mathrm{CP}$ polarisation. Examining Fig. 6 again, the outgoing wave is 
Right-Hand Circularly Polarised (RHCP) in the frequency range $1.5 \mathrm{GHz}$ to point $A(2.812 \mathrm{GHz})$, Elliptically Polarised (EP) in the range $A$ to $L 1(3.042 \mathrm{GHz})$ Linearly Polarised (LP) at $L 1$. The outgoing wave is EP in the range $L 1$ to $C 1$ (3.17 $\mathrm{GHz})$, while in the range $C 1$ to $C 2(3.358 \mathrm{GHz})$ it is $L H C P$. The resonant frequency point $\mathrm{Cm}(3.326 \mathrm{GHz})$ gives a marginally LHCP outgoing wave. The axial ratio of the surface for the ingoing and outgoing waves is depicted in Fig. 8.

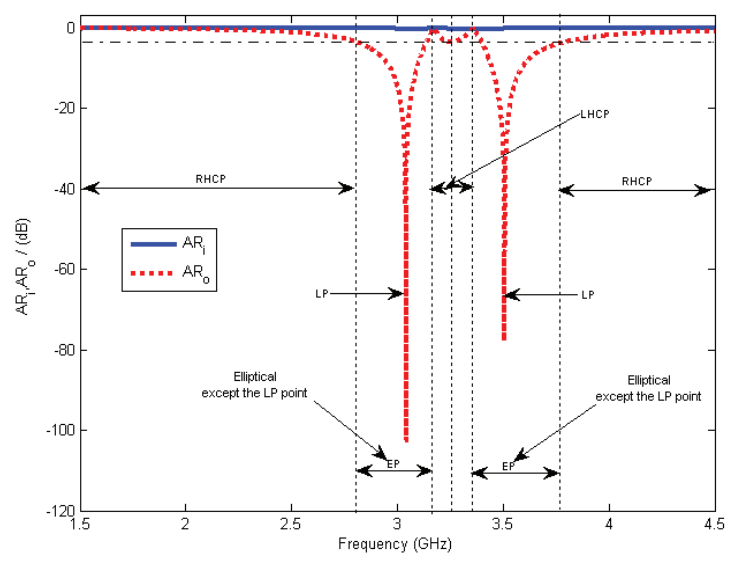

Fig. 8 Axial ratio for the outgoing and ingoing waves

A summary of the results is tabulated in Table 1. where $f_{r, x}$ and $f_{r, y}$ are the resonant frequency for the $x$-and $y$-polarised components of the plane wave respectively, and $f_{180}$ is the frequency at which the phase difference is approximately $180^{\circ}$.

TABLE I PARAMETRIC ANALYSIS OF THE EBG WITH SHEET VIA

\begin{tabular}{c|ccc|ccc|cc}
$\boldsymbol{f}$ in & \multicolumn{3}{|c|}{ Length (mm) } & \multicolumn{3}{c|}{ Thickness (mm) } & \multicolumn{2}{c}{ Angle (deg) } \\
$\mathbf{G H z}$ & $\mathbf{2}$ & $\mathbf{8}$ & $\mathbf{1 2}$ & $\mathbf{0 . 0 5}$ & $\mathbf{1}$ & $\mathbf{2}$ & $\mathbf{5}$ & $\mathbf{4 5}$ \\
\hline$f_{r, x}$ & 2.96 & 3.32 & 3.74 & 3.18 & 3.27 & 3.32 & 3.19 & 3.25 \\
$f_{r, y}$ & 2.96 & 3.03 & 3.08 & 2.93 & 2.97 & 3.03 & 2.94 & 3.25 \\
$f_{180}$ & ---- & 3.15 & 3.11 & 3.05 & 3.10 & 3.16 & 3.05 & 3.25
\end{tabular}

The advantage of this particular EBG surface is that the reflection phase can be controlled in several different ways and also in certain bands different polarisation conversion behavior can be obtained.

\section{CONCLUSIONS}

In this paper an EBG surface with an inclined sheet via has been evaluated. It has been shown that in contrast to the traditional 'mushroom' EBG surface the inclined via surface exhibits a conversion property which can either be to a linear wave, or to a wave of a different-handed circular polarisation. The surface has this property due to its inherent anisotropy which influences the phase difference between the $x$ and $y$ components in the reflected wave. It has been observed in the parametric study that the phase difference between the reflection phases of the $x$ and $y$ components of the plane wave can be fine-tuned by varying three parameters (Table 1). The bandwidth exhibited by this PDEBG is $7.7 \%$, which is adequate for GPS applications, subjected to constraints on the size of the unit cell. There are potential applications for this surface including the use in simple-to-feed single-port antennas for GPS applications and low profile and multiband planar devices.

\section{REFERENCES}

[1] Zhang Guohua, Zhu Chang, Fu Yunqi, Yuan Naichang, "Using high impedance ground plane for radiation improving in microwave antennas", Microwave Conference Proceedings APMC 2005. AsiaPacific Conference Proceedings, Vol. 3, Page(s): 4 pp, 4-7 Dec. 2005.

[2] Duane Yunfeng, Liu Shaobin, Wang Yong, "A Novel Dual-band Compact Electromagnetic Bandgap (EBG) Structure and its Application", ICMMT 2008, Vol. 1, Page(s):51-53, 21-24 April 2008

[3] Yang, Rahmat-Samii, "Reflection phase characterizations of the EBG ground plane for low profile wire antenna applications", Antennas and Propagation, IEEE Transactions on, Vol. 5, Issue 10, Part 1, Page(s):2691-2703, Oct. 2003

[4] Yan, Qiang Gao, Wang, Zhu, Yuan, "A novel polarisation convert surface based on artificial magnetic conductor", Asia-Pacific Microwave Conference Proceedings, APMC 2005, Vol. 3, Page(s):2 pp 4-7 Dec. 2005.

[5] A. Aminian., Yang, Rahmat-Samii, "In-phase reflection and EM wave suppression characteristics of electromagnetic band gap ground planes", IEEE Antennas and Propagation Society International Symposium 2003, Vol. 4, Page(s): 430- 433, 22-27 June 2003.

[6] Rajo-Iglesias, Inclan-Sanchez, Vazquez-Roy, Garcia-Muoz, "Size Reduction of Mushroom-Type EBG Surfaces by Using Edge-Located Vias", IEEE Microwave and Wireless Components Letters, Vol: 17, Issue: 9, Page(s): 670-672, Sept. 2007.

[7] Yang, Rahmat-Samii, "Polarisation-dependent electromagnetic band gap (PDEBG) structures: Designs and applications", Microwave and Optical Technology Letters, Vol. 41, Issue. 6, Page(s): 439 - 444, June 2004.

[8] Yang, Rahmat-Samii, "A low profile single dipole antenna radiating circularly polarised waves", IEEE Transactions on Antennas and Propagation, Vol: 53, Issue: 9, page(s): 3083-3086, Sept. 2005

[9] Fusco and Simms, "Reflected circular polarisation conservation using textured surface", Electronics Letters, Vol: 43, Issue: 18, page(s): 962963, August 312007.

[10] Christopoulos, Goussetis, Feresidis, Vardaxoglou, "Metamaterials with multiband AMC and EBG properties", Microwave Conference, 2005 European, Vol: 1, page(s): 3, Page(s): 4-6, Oct. 2005.

[11] Yunqi Fu and Naichang Yuan, "surface-wave bandgap of Polarisation dependent Electromagnetic bandgap Structures", Microwave and Optical Technology Letters, Volume 49, Issue 4, Page(s): 946 - 949, 26 Feb 2007.

[12] Yang, Rahmat-Samii, "Wire antennas on artificial complex ground planes: a new generation of low gain antennas", IEEE Antennas and Propagation Society International Symposium, 2004, Vol: 1, Page(s): 309- 312, 20-25 June 2004.

[13] Rahmat-Samii, "Metamaterials in Antenna Applications: Classifications, Designs and Applications", IEEE International Workshop on Antenna Technology Small Antennas and Novel Metamaterials, 2006 , page(s): 1- 4, March 6-8, 2006.

[14] Metamaterials: Physics and engineering explorations, edited by Nader Engheta, Ziolkowski, IEEE press, John Willey \& sons Inc., 2006.

[15] P. B. Johns, "A symmetrical condensed node for the TLM method" IEEE Transactions on Microwave Theory and Technique, MTT-35, Page(s): 370-377, Apr. 1987. 